\title{
An Improved Pragmatic Approach to Coded Modulation Design with Multiple Carrier Frequencies
}

\author{
Ching-Hua Chuang and Lin-Shan Lee \\ Department of Electrical Engineering, Rm 521 \\ National Taiwan University \\ 106-17 Taipei, Taiwan, Republic of China \\ TEL: 886-2-783-8642, FAX: 886-2-782-4814 \\ e-mail: lsl@iis.sinica.edu.tw
}

\begin{abstract}
An improved pragmatic approach to coded modulation design which provides higher coding gains especially for noisy channels is proposed in this paper. The basic pragmatic approach previously proposed by Viterbi et al. is adopted, but integrated with the multiple carrier frequency concept. An optimum rate $\frac{1}{2}$ convolutional code is used to design a general coded modulation system which can be implemented easily. This new scheme is shown to perform much better than the original pragmatic approach at low to modest signal-to-noise ratio(SNR) conditions. It provides significant coding gains for practical channel conditions but at a price of slight loss in asymptotic coding gain(ACG). The system performances with respect to various design issues factors and parameters including the signal constellations, mapping rules, ACG's and minimum Euclidean distances are analyzed and illustrated in detail. Extensive simulation results also support the concept and demostrate the validity of the analysis.
\end{abstract}

\section{Introduction}

Trellis coded modulation(TCM) has been used as a very attractive bandwidth efficient technique for digital transmissions[1]. However, it is well known that the bursty error is inevitable for TCM, especially at noisy channels with low SNR. This is because the multi-branch unmerged paths very often have competible metric values with the correct path. For trellis codes with parallel transition$\mathrm{s}$, the number of single signal-error events also increases as well. This makes it relatively difficult to directly apply conventional TCM schemes in power-limited channels or noisy communication environments. A pragmatic design of encoder/modulator has been proposed by Viterbi et al.[2]. This design provides almost equivalent coding gains to the best Ungerboeck codes using an optimum rate $\frac{1}{2}, 64$. state binary convolutional code, which is VLSI implemented aboundingly. But the performance of this design also inevitably degrades at low SNR because the basic signal constellations used are not changed. In this paper, an improved encoder/modulator design with higher coding gains especially at noisy channels is therefore proposed based on the previous pragmatic design, but integrated with the concept of multiple carrier frequencies[3].

In the following, the backgrounds including the pragmatic approach to encoder/modulator design and the concep$t$ of multiple carrier frequencies are first very briefly re- viewed in the next section. In section 3 , the improved encoder/modulator design will be presented and explained in detail. The system performance analysis and simulation results are then given in section 4 and section 5 respectively. The concluding remarks are finally made in section 6 .

\section{Background Review}

\subsection{Pragmatic Approach to Encoder/ Modulator Design}

The pragmatic approach to encoder/modulator design[2] provides the means to achieve reasonable coding gains using a single existing good convolutional encoder and a very simple signal mapping only. The design issues are summarized below.

An "industry standard" rate $\frac{1}{2}, 64$-state binary convolutional encoder is used to encode one out of the $k-1$ information bits to obtain two coded bits to select one signal from a four-point sector $\{X 10, X 11, X 01, X 00\}$, which is taken from a total of $2^{k-2}$ sectors according to the other $k-2$ uncoded bits, where $X$ indicates the $k-2$ uncoded bits. Therefore, there are $2^{k-2}$ parallel transitions on each branch of the trellis structure. Figure 1 is an example of signal constellation for 8ASK $(k=3, M=8)$.

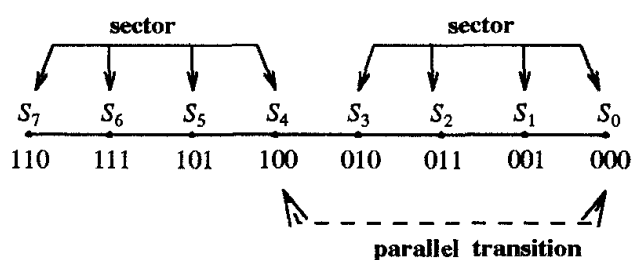

Figure 1: Signal constellation of a pragmatic design for coded MASK scheme for $M=8$.

For the optimum code chosen, the ACG for transmission over additive white Gaussian noise(AWGN) channel can be obtained for a coded MASK scheme, for example, as follows[2],

$$
\mathrm{ACG} \geq 10 \log \left\{4 \cdot \frac{M^{2}-4}{M^{2}-1} \cdot \frac{7}{8}\right\}
$$




\subsection{Multiple Carrier Frequency Concept in Coded Modulation}

Coded frequency/phase modulation was first proposed by Padovani and Wolf[3]. In this technique, binary FSK and $\frac{M}{2}$-ary PSK schemes were combined to replace the conventional MPSK scheme in coded modulation. The two carrier frequencies are chosen as $\left(\omega_{c}+\frac{h \pi}{T}\right)$ and $\left(\omega_{c}-\frac{h \pi}{T}\right) \mathrm{rad} / \mathrm{s}$, where $h$ is the modulation index. The signal space for such schemes is four-dimensional in general. Using the orthonormal bases $\left\{\psi_{i}(t), i=1 \sim 4\right\}$ previously chosen by [3] to span the space, the signal constellation of $2 \mathrm{FSK} / 4 \mathrm{PSK}$ as shown in Figure 2, for example, can be represented as

$$
\begin{aligned}
S(t)= & \sqrt{\frac{2}{T}} \cos \left[\left(\omega_{c} t \pm \frac{h \pi}{T} t\right)-\phi_{i}\right], \quad 0 \leq t<T \\
\phi_{i} & \in\left\{0, \frac{\pi}{2}, \pi, \frac{3 \pi}{2}\right\}
\end{aligned}
$$

and the eight signal vectors are given by

$$
\begin{array}{cccc}
S_{0}: & (1,0,0,0) ; & S_{1}: & \left(c_{1}, c_{2}, \sqrt{D}, 0\right) \\
S_{3}: & (0,1,0,0) ; & S_{2}: & \left(-c_{2}, c_{1}, 0, \sqrt{D}\right) \\
S_{4}: & (-1,0,0,0) ; & S_{5}: & \left(-c_{1},-c_{2},-\sqrt{D}, 0\right) \\
S_{7}: & (0,-1,0,0) ; & S_{6}: & \left(c_{2},-c_{1}, 0,-\sqrt{D}\right)
\end{array}
$$

where

$$
c_{1}=\frac{\sin 2 \pi h}{2 \pi h} ; c_{2}=\frac{1-\cos 2 \pi h}{2 \pi h} ; \quad D=1-c_{1}^{2}-c_{2}^{2}
$$

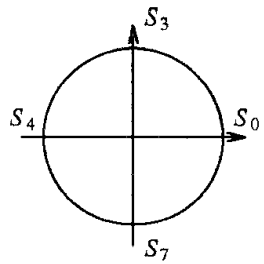

Figure 2: Signal constellation of 2FSK/4PSK scheme.

The same concept can be applied to combine 2FSK and $\frac{M}{2}$-ary ASK modulation. For example, the signal constellation of $2 \mathrm{FSK} / 4 \mathrm{ASK}$ scheme as shown in Figure 3 can be represented as

$$
\begin{aligned}
S_{0,1,4,5}(t) & =\left( \pm \frac{1}{2}, \pm \frac{3}{2}\right) \triangle \sqrt{\frac{2}{T}} \cos \left(\omega_{c} t+\frac{h \pi}{T} t\right) \\
S_{6,7,2,3}(t) & =\left( \pm \frac{1}{2}, \pm \frac{3}{2}\right) \triangle \sqrt{\frac{2}{T}} \cos \left(\omega_{c} t-\frac{h \pi}{T} t\right)
\end{aligned}
$$

where $\triangle$ is the Euclidean distance between the neighboring signals at the same carrier frequency. After normalizing the average signal energy $\bar{E}_{s}$ to unity $\left(\triangle^{2}=0.8\right)$, the signal vectors are given by

$$
\begin{aligned}
& S_{0,1,4,5}=( \pm 0.4472, \pm 1.3416)(1,0,0,0) \\
& S_{6,7,2,3}=( \pm 0.4472, \pm 1.3416)\left(c_{1}, c_{2}, \sqrt{D}, 0\right)
\end{aligned}
$$

Apparently two vectors instead of four will be enough to represent these signals. This is useful in reducing the number of matched filters in receiver design.

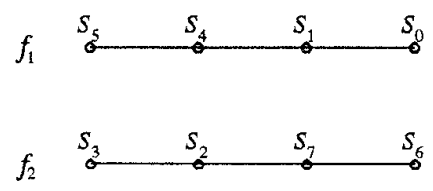

Figure 3: Signal constellation of 2FSK/4ASK scheme.

\section{The Proposed New Scheme}

The encoder/modulator of the proposed new scheme is shown in Figure 4 (a) and (b) when coded $2 \mathrm{FSK} / \frac{M}{2}$-ASK or coded $2 \mathrm{FSK} / \frac{M}{2}-\mathrm{PSK}$ is used, respectively. The basic structure is very similar to the original pragmatic approach. One out of the $k-1$ information bits is encoded by the optimum convolutional encoder with rate $\frac{1}{2}$ and 64 states. The two generating polynomials producing the two coded bits $b_{0}, b_{1}$ are $G_{0}(D)=1+D+D^{2}+D^{3}+D^{6}$, and $G_{1}(D)=1+D^{2}+D^{3}+D^{5}+D^{6}$ respectively[4]. Parallel transitions apparently exist in the trellis structure for $M=2^{k} \geq 8$. In order to simplify the mapping rules of coded bits to channel signals, we use a specific bit to selec$t$ one of the two carrier frequencies $f_{1}, f_{2}$, and it is found that the Euclidean distances between parallel transitions will be maximized when the second bit, $b_{1}$, is used for coded $2 \mathrm{FSK} / \frac{M}{2}$-ASK scheme, while the least significant bit, $b_{0}$ for coded $2 \mathrm{FSK} / \frac{M}{2}$-PSK scheme. As it is shown in Figure 4 (a) and (b), they are marked with an "F" and an "X" respectively. This is because the signals associated with parallel transitions with maximum distances on the constellation belong to the same carrier frequency. The two coded bits $b_{0}$ and $b_{1}$ are then used to select one signal from the fourpoint sector, which is in turn selected by the other uncoded bits. The rest of mapping rules for assigning bit patterns to signals are summarized below.

1. In order to minimize the bit error probability, the assignment of uncoded bits to signals associated with parallel transitions should be in such a way that bit patterns with smaller number of different bits are assigned to signals with smaller Euclidean distances.

2. For exactly the same reason as above, the assignment of the two coded bits to one signal in the four-point sector should also be in such a way that $(10,01)$ or $(11,00)$ are mapped to the signals with larger distances while $(10,11)$ or $(01,00)$ to those with smaller distances and so on[2].

3. Another mapping rule in fact depends on the modulation index $h$. For example, in the signal constellation of Figure $3, d^{2}\left(S_{1}, S_{7}\right) \geq d^{2}\left(S_{1}, S_{2}\right)$ when $h \geq 0.5$, but this situation is reversed, i.e., $d^{2}\left(S_{1}, S_{7}\right)<\overline{d^{2}}\left(S_{1}, S_{2}\right)$, when $h<0.5$. The assignment of the two coded bits to signals $S_{2}$ and $S_{7}$ given the bit pattern assignmen$\mathrm{t}$ of $S_{1}$ should therefore consider such dependence of distance on modulation index $h$. The mapping shown in Figure 4 is actually based on the assumption that $h \geq 0.5$.

In this situation of Figure 4, it can be easily seen that only $k-1$ gates are need to perform the XOR operation between the frequency bit and the others respectively in choosing the amplitudes transmitted for $\frac{M}{2}-\mathrm{ASK}$, and even none is needed in choosing the phases for $\frac{M}{2}$-PSK. 


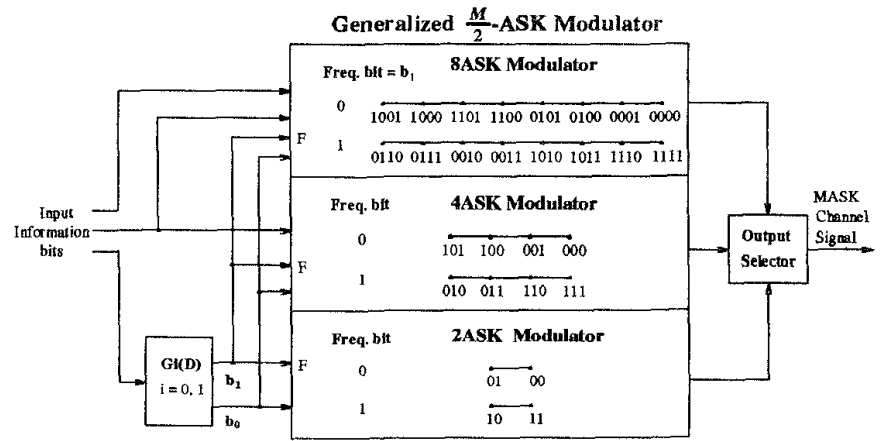

(a)

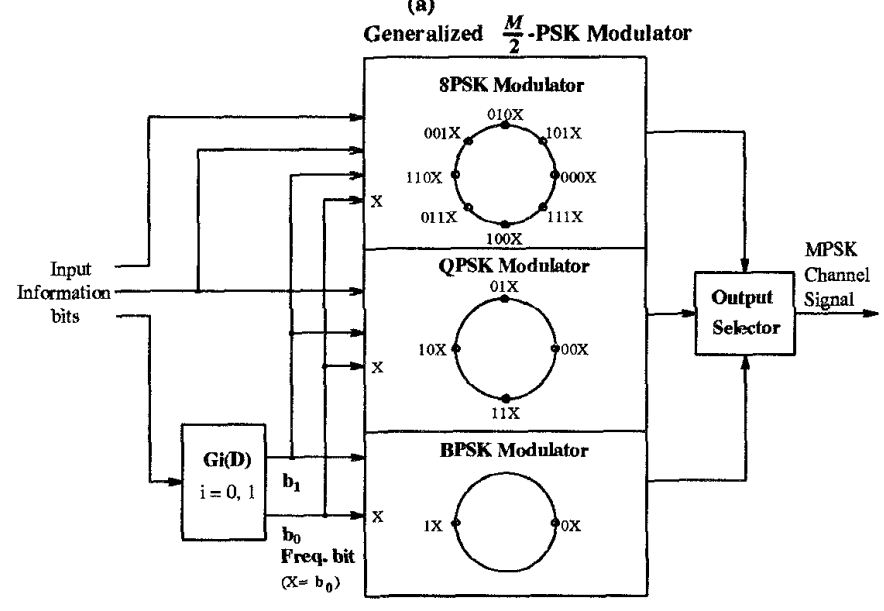

(b)

Figure 4: The encoder/modulator for the proposed new scheme with (a) coded 2 FSK $/ \frac{M}{2}-\mathrm{ASK}$, and (b) coded 2 FSK $/ \frac{M}{2}$-PSK for $M=4,8,16$.

\section{System Performance Analysis}

Free distance $d_{f r e e}$ and the minimum squared Euclidean distance $\Delta_{0}^{2}$ between any two signals are two important parameters in estimating the system performance in TCM design. The former is lower bounded by the minimurn value of Euclidean distances for single signal-error events and multi-branch unmerged paths if parallel transitions exist, while the latter is decided by the selected signal constellation. These two parameters for the proposed new schemes have been analyzed in detail. In the following, analysis for coded 2 FSK $/ \frac{M}{2}$-ASK will be presented as an example, but the results for coded 2 FSK $/ \frac{M}{2}$-PSK will also be provided as well.

Here we consider coded 2 FSK/ $\frac{M}{2}$-ASK only. Because the MASK signals have unequal energy, normalization of the squared Euclidean distance with respect to average signal energy $\bar{E}_{s}$ is necessary. In fact here $\bar{E}_{s}$ is further normalized to unity for simplicity so that it suffices to compare $d_{f r e e}^{2}$ only. The minimum normalized squared Euclidean distance (NSED) between the pair of nearest neighbors within the same parallel-transition bundled branches obviously cquals to $4 \triangle^{2}$.

On the other hand, for any rate $\frac{1}{2}$ binary convolutional code with given constraint length achieving maximum free Hamming distance, $d_{f}$, the first and last unmerged branch pairs always differ from the corresponding branches for correct path in both the two coded bits. Because the minimum Euclidean distance of the branch associated with two Hamming weights is less than two times that of the branch associated with one Hamming weight, the worst case is that each pair of non-identical branches differs in both the two coded bits (this is not exactly true for coded 2 FSK $/ \frac{M}{2}$-PSK for some values of $M$ and modulation index $h$ ). Therefore, the NSED between multi-branch unmerged paths is lower bounded by

$$
\mathrm{NSED} \geq \frac{d_{f}}{2}\left\{\frac{\triangle^{2}}{2}\left(1-c_{1}\right)\right\} \quad M \geq 4, h \geq 0.5
$$

And the free NSED is lower bounded by

$$
d_{f r e e}^{2} \geq \min \left\{4 \triangle^{2}, \frac{d_{f}}{2}\left[\frac{\triangle^{2}}{2}\left(1-c_{1}\right)\right]\right\} \quad M \geq 8, h \geq 0.5
$$

Because the minimum Euclidean distance between equally spaced signals for the uncoded $\frac{M}{2}-\mathrm{ASK}$ is also $\triangle$, the $A C G$ is given as

$$
\mathrm{ACG} \geq 10 \log \left\{\min \left[4, \frac{d_{f}}{4}\left(1-c_{1}\right)\right]\right\} \quad M \geq 8, h \geq 0.5
$$

For the code we used, $d_{f}=10$,

$$
\mathrm{ACG} \geq 10 \log \left\{\min \left[4, \frac{5}{2}\left(1-c_{1}\right)\right]\right\} \quad M \geq 8, h \geq 0.5
$$

For $M=4$, there is no parallel transition and thus the ACG is lower bounded by the minimum NSED between multi-branch unmerged paths.

Let the minimum Euclidean distance between equally spaced signals for the coded MASK scheme is $\triangle^{\prime}$, the average signal energies of the two coded schemes are given as

$$
\bar{E}_{s}= \begin{cases}\left(\triangle^{\prime}\right)^{2} \cdot \frac{M^{2}-1}{12} & \text { Coded MASK scheme } \\ (\triangle)^{2} \cdot \frac{(M / 2)^{2}-1}{12} & \text { Coded 2FSK } / \frac{M}{2} \text {-ASK scheme }\end{cases}
$$

For $\bar{E}_{s}$ being normalized to unity, one has

$$
\left(\triangle^{\prime}\right)^{2}=\frac{12}{M^{2}-1} ; \quad(\triangle)^{2}=\frac{48}{M^{2}-4}
$$

The parameter $\triangle_{0}^{2}$ of the coded MASK scheme is obviously equal to $\triangle^{\prime 2}$. For the parameter $\triangle_{0}^{2}$ of the coded $2 F S K / \frac{M}{2}$ ASK scheme, we need to consider the NSED between the nearest signals at different carrier frequencies. It is found that the parameter $\Delta_{0}^{2}$ is equal to $\frac{\Delta^{2}}{2}\left(1+c_{1}\right)$ for coded $2 \mathrm{FSK} / \frac{M}{2}$-ASK for $h \geq 0.5$.

The analysis results for coded 2 FSK $/ \frac{M}{2}$-ASK and coded 2FSK/ $\frac{M}{2}$-PSK are summarized in Tables 1, 2 respectively for $M \stackrel{2}{=} 4,8,16$. The numbers in parentheses in the right column of the tables for $\triangle_{0}^{2}$ are the improvements of coded 2 FSK $/ \frac{M}{2}$-ASK(PSK) with respect to coded MASK(MPSK) in terms of numbers of $d B$, i.e.,

$$
10 \log \frac{\triangle_{0}^{2} \text { of coded } 2 \text { FSK } / \frac{M}{2} \text {-ASK (PSK) }}{\triangle_{0}^{2} \text { of coded MASK (MPSK) }}
$$

For the data for coded 2 FSK $/ \frac{M}{2}$-ASK as shown in the left column of ACG values in Table 1, except that coded 
2FSK/2ASK at $h=0.75$ has $0.35 d B$ of improvement in ACG, the new scheme in general has degraded ACG. This is in fact a very natural result because we have adopted the most conservative estimation of the minimum NSED value for multi-branch unmerged paths which consist of branches with two Hamming weights only. However, in Table 1, one can see that the proposed new coded 2 FSK $/ \frac{M}{2}-A S K$ scheme always provides larger $\triangle_{0}^{2}$, with an improvement of 2-4 $d B$ with respect to coded MASK. This is very helpful in reducing the decoding errors due to given truncation length for the Viterbi decoder, as will be verified by the simulation results discussed below.

For the coded $2 \mathrm{FSK} / \frac{M}{2}$-PSK scheme, we can see in the left column of Table 2 that in general the coded $2 \mathrm{FSK} / \frac{M}{2}$ PSK achieves the same ACG as the coded MPSK scheme for $M>8$ because both of them are decided by the minimum Euclidean distance for the same signals associated with parallel transitions, but on the other hand the proposed coded 2 FSK $/ \frac{M}{2}$-PSK scheme provides improved $\triangle_{0}^{2}$ (0.9-5.8 $\mathrm{dB}$ ) with respect to the coded MPSK scheme for $M \geq 8$. As for the coded QPSK $(M=4)$, because $d_{f r e \varepsilon}$ is lower bounded by the multi-branch unmerged paths and $\triangle_{0}^{2}$ is better than the coded 2 FSK/2PSK, the latter is not recommended but listed here for reference purpose only. Some recent work also studied the possible improvements obtainable by carefully adjusting the twisted phase angle (here is assumed to be $0^{\circ}$ ) between the corresponding signals at the two carrier frequencies $[3,5]$, which is also a possible direction for further analysis. All the above analysis results indicate that the new proposed scheme has a better ability in noise immunity such that it is especially suitable at low SNR conditions. This will be demonstrated by simulation results discussed below.

\section{Simulation Results}

In practical situation, the actual coding gain is often more useful than the ACG. Computer simulation has been performed for the coded 2 FSK $/ \frac{M}{2}-\mathrm{ASK}$ (PSK) and coded MASK(MPSK) schemes over the AWGN vector channels with the two-sided noise spectral density being $N_{o} / 2$, and the average energy per bit is set to be $E_{b}$. The results are plotted in Figures 5 and 6.

The simulation results for the coded 2 FSK $/ \frac{M}{2}$-ASK ( $h$ $=0.5$ ), coded MASK, and the exact BER's of the uncoded $\frac{M}{2}$-ASK for $M=4,8,16$ are plotted in Figure 5 . It is clear that the performance of the original pragmatic approach to encoder/modulator design inevitably degrades at low S$\mathrm{NR}$, while the new proposed scheme provides significant improvements in these situations. The coded $2 \mathrm{FSK} / 2 \mathrm{ASK}$ always has better performance than the coded 4ASK. The higher coding gain at lower SNR is apparent, and the improvement remains at about $1.36 d B$ even at a BER of $10^{-5}$. The much larger values of $\triangle_{0}^{2}$ in Table 1 explain this phenomenon. Figure 5 also clearly shows that the new proposed scheme provides an actual coding gain of about $2.24 d B$ than the coded MASK at a BER of $10^{-5}$, and still keeps satisfactory performance when the channel becomes noisy. Although the original coded MASK has good performance at high SNR, its actual coding gain degrades rapidly when SNR becomes lower. But the the proposed coded $2 \mathrm{FSK} / \frac{M}{2}$-ASK degrades much slower. For examples, when the $\frac{E_{b}}{N_{0}}$ value decreases from $10.5 d B$ to $7.5 d B$, the BER for the coded $8 \mathrm{ASK}$ increases from the order of $10^{-6}$ to the order of $10^{-2}$, while the coded $2 \mathrm{FSK} / 4 \mathrm{ASK}$ can maintain a performance on the order of $10^{-5}$. When the $\frac{E_{b}}{N_{0}}$ value decreases from $14.5 d B$ to $11.5 d B$, the BER for the coded $16 \mathrm{ASK}$ drops dramatically from the order of $10^{-6}$ to the order of $10^{-2}$, while the coded $2 \mathrm{FSK} / 8 \mathrm{ASK}$ does sustain the level of $10^{-5}$. Figure 6 shows the simulation results for the coded $2 \mathrm{FSK} / \frac{M}{2}$-PSK $(h=0.5,0.75)$, coded MPSK, and the exact BER's of the uncoded $\frac{M}{2}$-PSK for $M=4$, 16. We can see very similar performance behaviors as those in Figure 5 except that the coded QPSK has better performance than the coded 2FSK/2PSK, but this situation has been discussed in the previous section. In Figure 6, we can see that the coded 2FSK/8PSK not only has a much more attractive performance at low SNR, but also is about 1.45 $d B$ better at $10^{-6}$ BER.

As shown also in Figure 6 is the effect of carefully choosing the modulation index $h$ on the system performance. An improvement of about $0.5 \mathrm{~dB}$, more or less, is obtainable if the modulation index was selected properly for the adopted signal constellation and the mapping rules for bit patterns to signals, although this also implies slightly different bandwidth expansion $[3]$.

\section{Conclusion}

A new pragmatic approach to encoder/modulator design based on the concept of multiple carrier frequencies is proposed in this paper. The performance at low to modest SNR can be significantly improved while the simple trellis structure can be preserved such that it can be implemented easily. The analysis and simulation results show that the new approach provides higher actual coding gains and is especially suitable to noisy channels. The new scheme also provides further design parameters such as the modulation index for the carrier frequency difference and bit pattern mapping for signal constellation, etc.

\section{References}

[1] G. Ungerboeck. Channel coding with multilevel/phase signals. IEEE Trans. Inform. Theory, vol. IT-28:pp. 5567, Jan. 1982.

[2] Andrew J. Viterbi, Jack K. Wolf, Ephraim Zehavi, and Roberto Padovani. A pragmatic approach to trelliscoded modulation. IEEE Commun. Mag., vol. 25:pp. 11-19, July. 1989.

[3] Roberto Padovani and Jack K. Wolf. Coded phase/frequency modulation. IEEE Trans. Commun., vol. COM-34:pp. 446-453, May. 1986.

[4] J. Gonan. The weight spectra of some short low rate convolutional codes. IEEE Trans. Commun., vol. COM32:pp. 1050-1053, Sept. 1984.

[5] Aminuddin Chalid, Iwao Sasase, Hiroyuki Yashima, and Shinsaku Mori. Coded nonuniform phase/frequency modulation. THE TRANSACTIONS OF THE IEICE, vol. E 72:pp. 903-911, Aug. 1989. 
Table 1: Comparison of performance factors for coded MASK and $2 \mathrm{FSK} / \frac{M}{2}-\mathrm{ASK}$

\begin{tabular}{|c|c|c|c|c|c|c|c|}
\hline \multirow[b]{2}{*}{ M } & \multirow{2}{*}{$\begin{array}{l}\text { Modulation } \\
\text { scheme }\end{array}$} & \multicolumn{3}{|c|}{ ACG $(d B)$ over uncoded scheme } & \multicolumn{3}{|c|}{$\triangle_{0}^{2}\left(\bar{E}_{s}=1.0\right)$} \\
\hline & & $h=0.5$ & $h=0.75$ & $h=1.0$ & $h=0.5$ & $h=0.75$ & $h=1.0$ \\
\hline \multirow[b]{2}{*}{4} & coded 4ASK & \multicolumn{3}{|c|}{4.47} & \multicolumn{3}{|c|}{0.80} \\
\hline & coded 2F/2ASK & 3.98 & 4.82 & 3.98 & $2.0(3.98 d B)$ & $1.576(2.96 d B)$ & $2.0(3.98 d B)$ \\
\hline \multirow{3}{*}{8} & coded $8 \mathrm{ASK}$ & \multicolumn{3}{|c|}{5.23} & \multicolumn{3}{|c|}{0.190} \\
\hline & coded 2F/4ASK & 3.98 & 4.82 & 3.98 & $0.40(3.23 d B)$ & $0.315(2.20 \mathrm{~dB})$ & $0.40(3.23 d B)$ \\
\hline & coded 16ASK & \multicolumn{3}{|c|}{5.39} & \multicolumn{3}{|c|}{0.047} \\
\hline 16 & coded 2F/8ASK & 3.98 & 4.82 & 3.98 & $0.095(3.06 d B)$ & $0.075(2.03 d B)$ & 0.095 ( \\
\hline
\end{tabular}

Table 2: Comparison of performance factors for coded MPSK and 2 FSK $/ \frac{M}{2}$-PSK

\begin{tabular}{|c|c|c|c|c|c|c|c|}
\hline \multirow[b]{2}{*}{ M } & \multirow{2}{*}{$\begin{array}{c}\text { Modulation } \\
\text { scheme }\end{array}$} & \multicolumn{3}{|c|}{ ACG $(d B)$ over uncoded scheme } & \multicolumn{3}{|c|}{$\triangle_{0}^{2}\left(E_{9}=1.0\right)$} \\
\hline & & $h=0.5$ & $h=0.75$ & $h=1.0$ & $h=0.5$ & $h=0.75$ & $h=1.0$ \\
\hline \multirow[b]{2}{*}{4} & coded QPSK & \multicolumn{3}{|c|}{6.99} & \multicolumn{3}{|c|}{2.0} \\
\hline & coded 2F/2PSK & 3.98 & 4.82 & 3.98 & $2.0(0.0 d B)$ & $1.576(-1.02 d B)$ & $2.0(0.0 \mathrm{~dB})$ \\
\hline \multirow[b]{2}{*}{8} & coded 8PSK & \multicolumn{3}{|c|}{3.0} & \multicolumn{3}{|c|}{0.586} \\
\hline & coded $2 F / 4$ PSK & 2.6 & 3.0 & 3.0 & $0.727(0.94 d B)$ & $1.576(4.30 \mathrm{~dB})$ & $2.0(5.33 d B)$ \\
\hline & coded 16PSK & \multicolumn{3}{|c|}{5.33} & \multicolumn{3}{|c|}{0.152} \\
\hline 16 & coded 2F/8PSK & 5.33 & 5.33 & 5.33 & $0.586(5.85 \mathrm{~dB})$ & $0.586(5.85 \mathrm{~dB})$ & $0.586(5.85 d B)$ \\
\hline
\end{tabular}

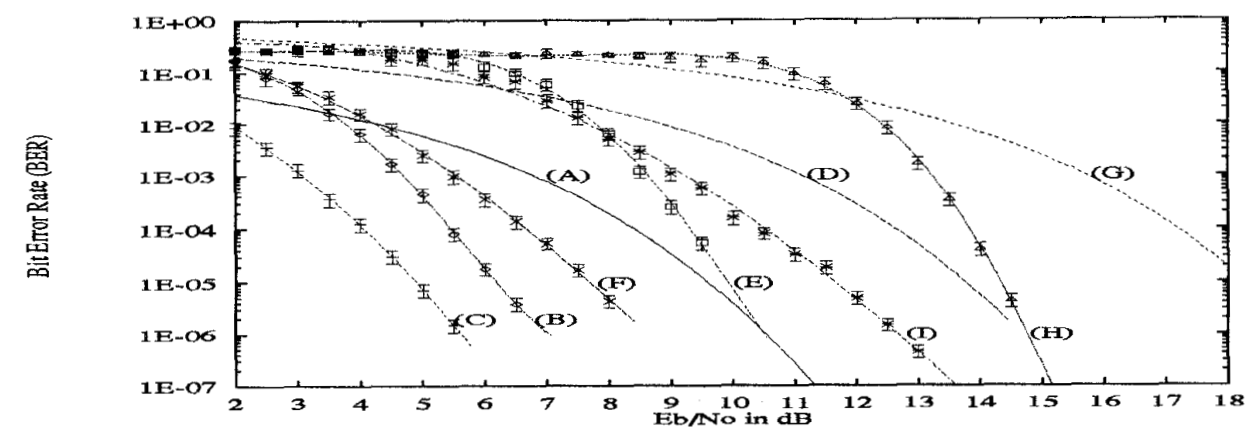

Figure 5: BER for (A) : uncoded 2ASK, (B) : coded 4ASK, (C) : coded 2FSK/2ASK at $h=0.5$, (D) : uncoded 4ASK, (E) : coded $8 \mathrm{ASK},(\mathrm{F}):$ coded $2 \mathrm{FSK} / 4 \mathrm{ASK}$ at $h=0.5,(\mathrm{G}):$ uncoded $8 \mathrm{ASK},(\mathrm{H}):$ coded $16 \mathrm{ASK},(\mathrm{I}):$ coded $2 \mathrm{FSK} / 8 \mathrm{ASK}$ at $h$ $=0.5$.

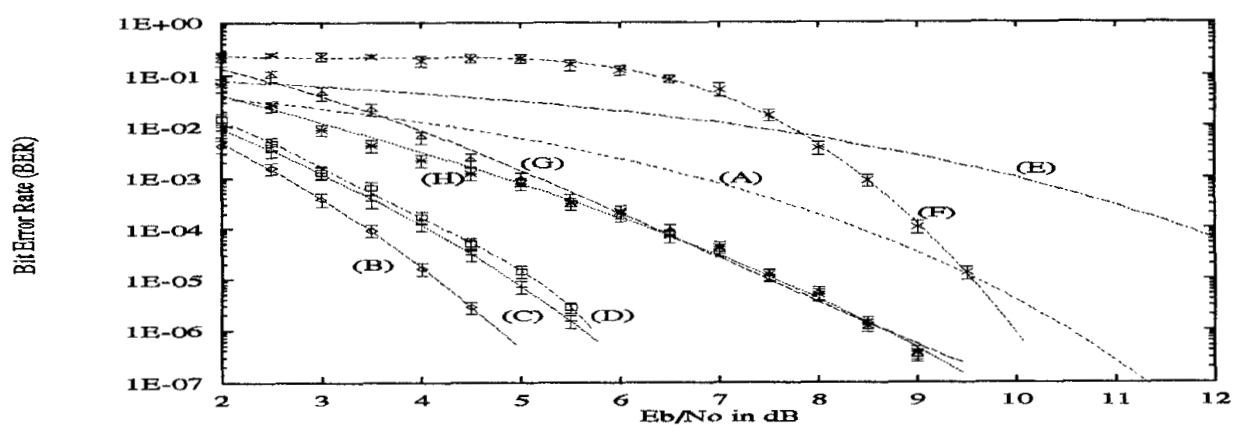

Figure 6: BER for (A) : uncoded 2PSK, (B) : coded 4PSK, (C) : coded 2FSK/2PSK at $h=0.5$, (D) : coded 2 FSK/2PSK at $h=0.75,(\mathrm{E}):$ uncoded 8PSK, (F) : coded 16PSK, (G) : coded 2 FSK $/ 8$ PSK at $h=0.5,(\mathrm{H}):$ coded 2 FSK $/ 8$ PSK at $h=$ 0.75 . 